\title{
Probing the relaxation towards equilibrium in an isolated strongly correlated 1D Bose gas
}

Authors: Stefan Trotzky, Yu-Ao Chen, Andreas Flesch, Ian P. McCulloch, Ulrich Schollwöck, Jens Eisert, Immanuel Bloch

arXiv:1101.2659

\section{Recommended with a Commentary by Leonid Glazman, Yale University.}

In statistical mechanics, one assumes that a generic macroscopic system, even being isolated from the rest of the world, would eventually reach a local thermal equilibrium. Density matrix of a finite-size part of such system as long as that part comprises many particles, would reach the Gibbs distribution. The parameters of the equilibrium distribution are fixed by the additive conserving quantities (particle number, energy, momentum, etc.). Normally, we expect the approach to equilibrium to be controlled by a spectrum of relaxation rates found from an appropriate kinetic equation [1]. However, there are prominent counter-examples to that common wisdom.

The approach to thermal equilibrium of a system of interacting particles, in any dimension, may be hindered by disorder, resulting in a "many-body localization". That possibility was raised in Ref. [2] and received considerable attention recently, see Ref. [3] and references therein. Abundance of integrals of motion in a disorder-free system also deemed to prevent thermalization. Such a possibility is foreseen in quantum integrable one-dimensional systems [4]. Quite remarkably, experimental investigation of the latter subtle roadblock to equilibration became possible in the context of cold gases [5]. Violation of integrability is expected to open channels for relaxation. That apparently what the recent work of Trotzky et. al. [6] shows.

The article of Trotzky et al [6] describes the measurement and a numerical simulation of the relaxation processes in a one-dimensional Bose gas subject to a potential of an optical lattice. A single one-dimensional system in the experiment [6] comprised of about $N=30$ atoms of ${ }^{87} \mathrm{Rb}$. Evolution of local density and current, and quasi-momentum distribution were investigated.

To study the evolution of density, an initial state in which every other site of a lattice was occupied was prepared. Initially, the lattice potential was 
high enough to prevent atoms from leaving their sites. Then, the potential was suddenly made lower to allow the tunneling. The occupation of initially empty sites was measured as a function of time. Measurements revealed strongly damped oscillations of the occupation (3 to 4 periods) on the way to the steady-state occupation of $1 / 2$. Apparently the decay was happening faster than one expects in the trivially-integrable limits of free or hard-core bosons (in either limit, the on-site density would follow the behavior of 0 -th order Bessel function). The authors view the enhanced relaxation as one of the major questions posed by their experiment.

The evolution of local currents was studied by observing the phase and amplitude of oscillations of the difference of occupation of two neighboring sites (odd-even or even-odd pairs). That apparently revealed a power-law decay $\left(\alpha t^{-\alpha}\right)$ of the amplitude $A$ with an exponent $\alpha \approx 0.86$. The on-site repulsion $U$ was on the order or larger than the tunnel coupling energy $J$, and its specific value did not affect $\alpha$ which was manifestly different from $\alpha=1 / 2$ expected in the two mentioned above integrable cases. The origin of the observed dependence, $A \propto t^{-0.86}$, remains unclear. It is emphasized in Ref. [6] that the observations are not explained by a mean-field theory or by rate equations.

Finally, experiments also addressed the time evolution of the off-diagonal (in site representation) components of the particle density operator. The observed behavior of that quantity (referred to as coherence) in the limits of $U / J \ll 1$ and $U / J \gg 1$ agreed with expectations from the free-particle evolution analysis. The qualitative behavior of the coherence at an arbitrary interaction was similar to that found in the two limits.

This work demonstrated a fast relaxation to a steady state, which is hard to distinguish from an equilibrium one. Yet, the way the investigated onedimensional system was approaching the steady state seem puzzling and quite different from the expectations based on the Markovian or on the mean-field dynamics.

1. K. Huang, Statistical Mechanics, 512pp., Wiley, 1987.

2. P. W. Anderson, Phys. Rev. 109, 1492 (1958).

3. Arijeet Pal, David A. Huse, Phys. Rev. B 82, 174411 (2010)

4. Bill Sutherland, Beautiful Models, World Scientific, 2004.

5. Toshiya Kinoshita, Trevor Wenger, and David S. Weiss, Nature, 440, 900 (2006).

6. Stefan Trotzky, Yu-Ao Chen, Andreas Flesch, Ian P. McCulloch, Ulrich 
Schollwöck, Jens Eisert, Immanuel Bloch, "Probing the relaxation towards equilibrium in an isolated strongly correlated 1D Bose gas", arXiv:1101.2659. 